\title{
Antidepressiva auch bei leichten Depressionen?
}

\begin{abstract}
? Wie kommt es, dass die Therapie der leichten Depression von den Experten noch immer kontrovers diskutiert wird?

Hegerl: Wie in den nachfolgenden Beiträgen von Herrn Prof. Lieb und Herrn Prof. Himmerich beschrieben, unterscheiden sich in der Tat die Empfehlungen verschiedener Leitlinien. Verwirrung entsteht schon allein durch die Unklarheit, wie eine "leichte Depression“ zu definieren ist. Zudem sind die relativ willkürlich gesetzten Definitionen der britischen Arzneimittel-Zulassungsbehörde NICE, was als klinisch bedeutsamer Behandlungseffekt angesehen wird („klinische Signifikanz"), unkritisch in die deutschen Leitlinien übertragen worden. Darüberhinaus wurden Befunde von Metaanalysen zur Behandlung der leichten Depression ohne ausreichend kritische Methodendiskussion übernommen.
\end{abstract}

\section{? Droht durch die Pharmakotherapie leichter Depressionen nicht} die Gefahr einer Überversorgung und Medikamentalisierung?

Hegerl: Nach wie vor ist davon auszugehen, dass nur eine Minderheit der depressiv Erkrankten eine optimale medikamentöse und/oder psychotherapeutische Behandlung erhält. Einer von zahlreichen Gründen hierfür ist auch die große Zurückhaltung der Menschen in Deutschland bezüglich Psychopharmaka. 80\% der Bevölkerung glauben, dass Antidepressiva süchtig machen und über $60 \%$, dass sie die Persönlichkeit verändern - alles unbegründete Befürchtungen. Meist werden valiumartige Beruhigungsmittel und Neuroleptika in einen Topf mit Antidepressiva geworfen. Bezeichnend ist, dass in einer Bevölkerungsumfrage in den 27 europäischen Ländern auf die Frage "Haben Sie in den letzten zwölf Monaten irgendwelche Antidepressiva eingenommen?" in Deutschland ca. 2,5\% mit "ja" antworteten. Dies ist die niedrigste Rate in allen 27 Ländern. Hierbei ist zudem zu bedenken, dass Antidepressiva oft auch zur Behandlung von Zwangsstörungen, Angsterkrankungen und teilweise auch bei Schlafstörungen eingesetzt werden. In Frankreich lag die Behandlungsrate beispielsweise bei 10\%, in England bei 8\%.
? Wie sollen diese Streitfragen letztlich entschieden werden? Hegerl: Es fehlen Studien, die die Frage beantworten, wieviel ein bisher unbehandelter Patient mit einer leichten Depression von einer konsequent durchgeführten Behandlung mit Antidepressiva profitieren würde. In diesen Studien müsste eine strikte Compliance-Kontrolle erfolgen und Patienten mit zahlreichen Vorbehandlungen müssten ausgeschlossen werden. Besonders unkritisch ist zurzeit die Interpretation von Ergebnissen aus Psychotherapiestudien. Da die Patienten nach Randomisierung wissen, ob sie der "richtigen Behandlungsgruppe" oder "nur der Kontrollgruppe" angehören, ist kein Placebo-, sondern nicht selten ein Noceboeffekt zu erwarten, wie dies im Rahmen der MIND-Studie (s. Beitrag von Himmerich) gezeigt und diskutiert wird. Insbesondere Wartegruppenkontrollen sind völlig ungeeignet und können zu Ergebnissen führen, die eine hohe Wirksamkeit vortäuschen, obwohl keine oder sogar negative Effekte der Behandlung vorliegen.

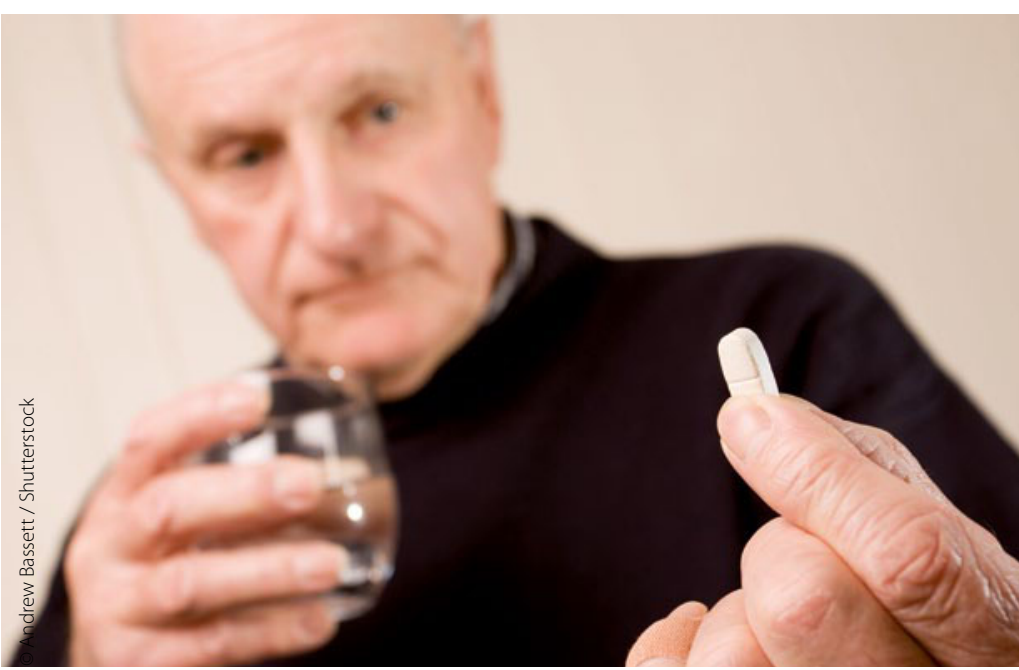

